\title{
PRELIMINARY SENSITIVITY STUDY OF AEROSOL LAYER HEIGHT FROM SYNTHETIC MULTIANGLE POLARIMETRIC REMOTE SENSING MEASUREMENTS
}

\author{
Weizhen Hou ${ }^{1,2}$, Haofei Wang ${ }^{1}$, Zhengqiang $\mathrm{Li}^{1, *}$, Lili Qie ${ }^{1}$, Bangyu Ge ${ }^{1}$, Cheng Fan ${ }^{1}$, Shuang Li $^{3}$ \\ ${ }^{1}$ State Environmental Protection Key Laboratory of Satellite Remote Sensing, Institute of Remote Sensing and Digital Earth, \\ Chinese Academy of Sciences, Beijing, China - (houwz, wanghf, lizq, qiell, geby, fancheng)@ radi.ac.cn \\ ${ }^{2}$ State Key Laboratory of Remote Sensing Science, Institute of Remote Sensing and Digital Earth, Chinese Academy of Sciences, \\ Beijing, China \\ ${ }^{3}$ Anhui Institute of Optics and Fine Mechanics, Chinese Academy of Sciences, Hefei, China - shli@aiofm.ac.cn
}

\section{Commission III, WG III/8}

KEY WORDS: Sensitivity Study, Aerosol Layer Height, Multiangle Polarimetric Remote Sensing, Information Content Analysis, Degree of Freedom for Signal

\begin{abstract}
:
Many previous studies have shown that multiangle, multispectral polarimetric remote sensing can provide valuable information on aerosol microphysical and optical properties, in which the aerosol layer height (ALH) is an important parameter but with less studies, especially in the near-ultraviolet (near-UV) and visible (VIS) wavelength bands. Based on the optimal estimation (OE) theory and information content analysis method, we focus on the sensitivity study of ALH with the synthetic data in the near-UV and VIS wavelength in the range of 410-865 nm, and further to assess the capability of multiangle intensity and polarization measurements for the retrieval of ALH. Unified Linearized Vector Radiative Transfer Model (UNL-VRTM) has been used as the forward model to simulate the intensity and polarized radiance at the top of atmosphere (TOA), as well as the Jacobians of TOA results with respective to corresponding parameters. The degree of freedom for signal (DFS) and a posteriori error are introduced to quantity the information content of ALH from the intensity and polarization measurements, respectively. By assuming the surface type, aerosol model, aerosol loads, prior errors and observation geometries, the sensitivity of ALH has been preliminarily investigated. The sensitivity study results show that the near-UV and polarization measurements are the important source of information content for the aerosol height retrieval in satellite remote sensing.
\end{abstract}

\section{INTRODUCTION}

Aerosol vertical distribution plays an important role in the study of radiative forcing, air quality, aerosol scattering in trace gas and ocean colour retrievals (Haywood and Boucher, 2000; Wang and Christopher, 2013; Butz et al., 2011; Gordon, 1997). For the active remote sensing, the aerosol vertical distribution can be effectively measured, such as the ground-based, airborne and space-borne measurements (Winker et al., 2010). While for the passive satellite remote sensing, the oxygen $\left(\mathrm{O}_{2}\right)$ A and $\mathrm{B}$ bands are usually used to study the aerosol vertical distribution based on synthetic data and real measurements (Sanghavi et al., 2012; Ding et al., 2014; Saders et al., 2015; Xu et al., 2017b, 2019). Many previous studies have shown that multiangle, multispectral polarimetric remote sensing can provide valuable information on aerosol microphysical and optical properties (Waquet et al., 2009; Hasekamp et al., 2011; Dubovik et al., 2011; Wu et al., 2015; Hou et al., 2018), in which the aerosol layer height (ALH) is one of important parameters but with less studies, especially in the near-ultraviolet (near-UV) and visible (VIS) wavelength bands (Wu et al., 2016).

The vertical distribution of aerosol is complex, which is not homogeneous due to the various structure depend on the height. The uncertainties from the treatment of the columnar atmosphere as homogeneous and independent as the height inevitably lead to the variations in aerosol optical features in model calculations $(\mathrm{Bi}$ et al., 2016). ALH is common used in the model calculation, while in the observational measurement, it is always instead of planetary boundary layer height (PBLH). The planetary boundary layer is the lowest layer of the atmosphere, which contains the vast majority of aerosols of the low troposphere. ALH is an important factor of atmospheric diffusion ability, which determines the vertical disperse range of pollutants emitted from the ground (Wang et al., 2019).

As the flagship of the environment and atmosphere observation satellite in the Chinese High-resolution Earth Observation System (CHEOS) program (Gu and Tong, 2015), Gaofen-5 (GF5) has been launched in May 2018, and the Directional Polarization Camera (DPC) is one of payloads (Li et al., 2018; Zheng et al., 2019). By inheritance the technology of Polarization and Directionality of the Earth's Reflectances (POLDER) (Deuzé et al., 2001), The DPC employed a charge coupled device (CCD) detection unit and realized spatial resolution of $3.3 \mathrm{~km}$ under a swath width of $1850 \mathrm{~km}$, which has integrated 3 polarized channels $(490,670,865 \mathrm{~nm})$ together with 5 non-polarized bands (443, 565, 763, 765, $910 \mathrm{~nm}$ ). Besides, the Multi-ViewingChannel-Polarization Imager (3MI) developed by EUMETSAT for the time-frame 2020-2040, will measure the radiance at the top of atmosphere (TOA) in 12 spectral channels, in which 9 channels will be polarized, including 410, 443, 490, 555, 670, 865, 1370, 1650, $2130 \mathrm{~nm}$ (Marbach et al., 2013).

In this paper, we focus on the preliminary sensitivity study of ALH with the synthetic data in the near-UV and VIS wavelength, and further to assess the capability of multiangle intensity and

\footnotetext{
* Corresponding author: lizq@ radi.ac.cn
} 
polarization measurements for the retrieval of ALH. For sensitivity study, the degree of freedom for signal (DFS) and a posteriori error of ALH have been employed. By assuming the surface type, aerosol model, aerosol loads, prior errors and multiviewing observation geometries, the sensitivity of ALH could be systematically analysed and discussed. In section 2 , we briefly describe the real aerosol vertical distribution based on the ground lidar measurements. Subsequently, we present the sensitivity study methodology in section 3 , and then conduct the analysis of information content based on these synthetic measurements in section 4. Finally, we give the conclusions in section 5.

\section{AEROSOL VERTICAL DISTRIBUTION}

The common measurements of ALH are taken by radiosonde, Sodar, microwave radiometer and Lidar, of which lidar is the effective way to retrieve the continue variation of the ALH (Collaud et al., 2014; Li et al., 2017). The Cloud-Aerosol Lidar with Orthogonal Polarization (CALIOP) has the capability to estimate the large-scale region ALH from space since 2006 (Winker et al., 2010). Meanwhile, similar measurements of the airborne Cloud Physics Lidar (CPL) has been utilized in many aircraft campaigns to profile the optical properties aerosol and cloud (McGill et al., 2002; Wu et al., 2016). Additionally, the ground-based lidar can provides more accurate measurements with high signal-to-noise ratio and time continuity (Wiegner et al., 2014; Tucker et al., 2009; Yang et al., 2013).

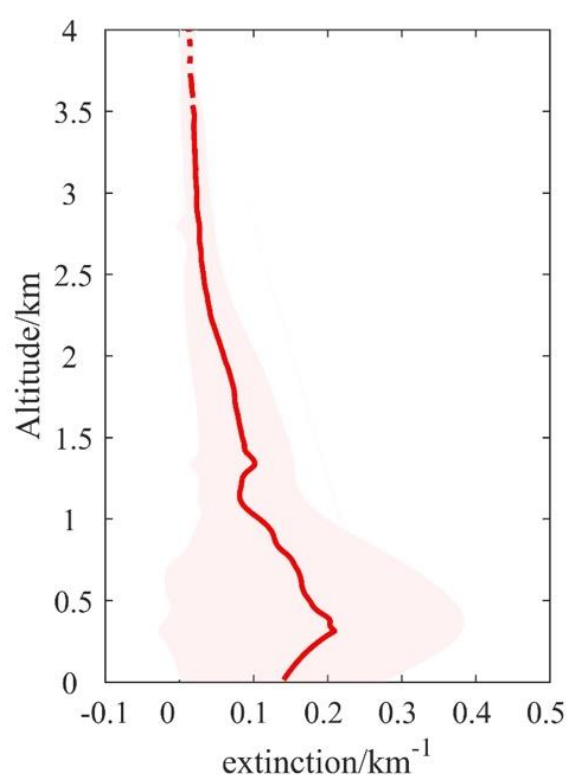

Figure 1. The mean extinction coefficients of aerosol in Oct. 6, 2017. The lines connected by red dots in the figure represent the average retrieved extinction coefficients, while the light red shadow indicates the standard deviation. The horizontal axis represents extinction coefficients and the vertical axis represents the attitude.

From the profile of aerosol extinction coefficients, we can clearly obtain the vertical distribution of aerosol. Based on the Fernald method, the aerosol extinction coefficient can be calculated (Fernald, 1984). Figure 1 shows the mean extinction coefficients of aerosol from a ground lidar called CE370 by CIMEL in France, which is located in the Institute of Remote Sensing and Digital Earth, Chinese Academy of Sciences in Beijing (Liu et al., 2017; Wang et al., 2019). We can see that the aerosol mainly exists in the height below $1 \mathrm{~km}$, where the extinction coefficients grows with a large gradient. At the height of $300 \mathrm{~m}$, it seemly the concentration gets the peak value. However, it should be noted that due to the insufficient overlap, the uncertainty of measurement below $200 \mathrm{~m}$ is large.

For lidar measurement, wavelet covariance transform is the most comment used method to identify ALH (Gamage and Hagelberg, 1993; Yang et al., 2013; Wang et al., 2019). The upper boundary of the AHL can be determined by the maximum variation gradient of the backscattering signal attenuated by aerosol (Seibert et al., 2000). From Figure 2, it is demonstrated that ALH starts to increase gradually from 08:00 a.m. and reaches its maximum at nearly 14: 00 p.m. From the midnight, ALH goes down rapidly and holds steady until the sunrise. Actually, the ALH shows a diurnal cycle from rising to decreasing along with the variation of the sunlight radiation.

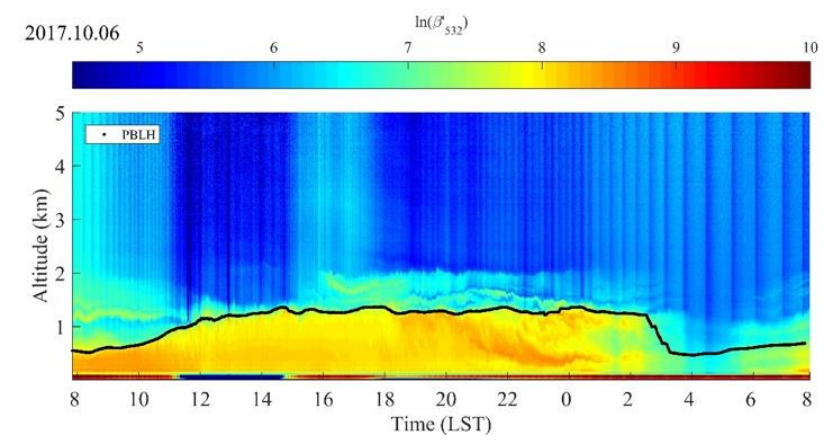

Figure 2. The aerosol layer height retrieved from wavelet covariance transform method in Beijing in Oct. 6, 2017. The

lines connected by black dots in the figure represent the

retrieved PBLH. The horizontal axis represents the local standard time (LST) and the vertical axis represents the height. Colorbar denotes the logarithm of the attenuated backscattering coefficient.

\section{METHODOLOGY}

\subsection{DFS and Posterior Error}

Following the optimal estimation (OE) theory (Rodgers, 2000; Dubovik et al., 2011), we have the forward model as

$$
\mathbf{y}=\mathbf{F}(\mathbf{x}, \mathbf{b})+\boldsymbol{\epsilon}
$$

where $\mathbf{x}$ is a state vector to be retrieved, the vector $\mathbf{b}$ contains these parameters that are not contained in $\mathbf{x}$ but quantitatively influence the TOA measurements, $\mathbf{y}$ is an observation vector, $\mathbf{F}$ means a forward model, and $\boldsymbol{\epsilon}$ means an experimental error term integrated by the observation noise and forward model uncertainty.

For the sensitivity study, the averaging kernel matrix can be represented as

$$
\mathbf{A}=\left(\mathbf{K}^{T} \mathbf{S}_{\epsilon}^{-1} \mathbf{K}+\mathbf{S}_{\mathrm{a}}^{-1}\right)^{-1} \mathbf{K}^{T} \mathbf{S}_{\epsilon}^{-1} \mathbf{K}
$$

where the superscript "-1" is the inverse operation of matrix, $\mathbf{S}_{\mathrm{a}}$ means the error covariance matrix of the a priori estimate $\mathbf{x}_{\mathrm{a}}$, the Jacobians matrix $\mathbf{K}$ corresponds to the partial derivatives of $\mathbf{F}(\mathbf{x})$ with respect to $\mathbf{x}$. Besides, $\mathbf{S}_{\epsilon}$ is the covariance matrix of the error from both the measurements and the forward model with

$$
\mathbf{S}_{\epsilon}=\mathbf{S}_{\mathrm{y}}+\mathbf{K}_{\mathrm{b}} \mathbf{S}_{\mathrm{b}} \mathbf{K}_{\mathrm{b}}^{T}
$$


in which $\mathbf{S}_{\mathrm{y}}$ is the measurement error covariance matrix, $\mathbf{S}_{\mathrm{b}}$ is the error covariance matrix for a vector $\mathbf{b}$ of forward model, and $\mathbf{K}_{\mathrm{b}}$ is the Jacobians matrix of measurements $\mathbf{y}$ with respect to the vector b (Frankenberg et al., 2012; Xu and Wang, 2015; Hou et al., 2018a, b).

The DFS of each individual retrieved parameter is equal to $\mathbf{A}_{i, i}$, and in the range of 0 and $1 . \mathbf{A}_{i, i}=1$ means that the observation is able to fully characterize the truth of $\mathrm{x}_{i}$, and $\mathbf{A}_{i, i}=0$ means that the observation do not contain any information on $\mathrm{x}_{i}$ at all. In other words, the closer the value of $\mathbf{A}_{i, i}$ to 1 , the better the retrieval of parameter $\mathrm{x}_{i}$. Meanwhile, a posteriori error covariance matrix $\widehat{\mathbf{S}}$ can be written as

$$
\widehat{\mathbf{S}}=\left(\mathbf{K}^{T} \mathbf{S}_{\epsilon}^{-1} \mathbf{K}+\mathbf{S}_{\mathrm{a}}^{-1}\right)^{-1},
$$

which means the statistical uncertainties in retrieved $\hat{\mathbf{x}}$ caused by measurement noise and the propagation of errors, and the posterior errors (absolute errors) are equal to the square root of diagonal elements (Hou et al., 2018a; Li et al., 2018).

\subsection{Forward Simulations and Parameter Settings}

To simulate the TOA measurements and corresponding Jacobian results in the near-UV and VIS wavelength from $410 \mathrm{~nm}$ to 865 nm of 3MI, Unified Linearized Vector Radiative Transfer Model (UNL-VRTM) is used as the forward model (Wang et al., 2014). UNL-VRTM integrates the VLIDORT code (Spurr et al., 2006), a linearized Mie code, a linearized T-Matrix code, a Rayleigh scattering module, line-by-line gas absorption calculation with HITRAN database, and some easy-used surface modules including the bidirectional reflectance distribution function (BRDF) and bidirectional polarized reflectance distribution function (BPDF). Based on UNL-VRTM, many studies have been carried to analyse the information content and develop the inversion algorithm for retrievals from the various measurements (Chen et al., 2017; Ding et al., 2016; Hou et al., 2016, 2017, 2018a, b; Xu and Wang, 2015; Xu et al., 2015, 2017a, b, 2018, 2019; Li et al., 2018; Zheng et al., 2019a, 2019b).

The kernel-driven BRDF model used in UNL-VRTM for surface reflectance can be written as

$$
\begin{gathered}
\rho_{s}\left(\mu_{0}, \mu_{v}, \phi, \lambda\right)=f_{\text {iso }}(\lambda)+k_{1}(\lambda) f_{\text {geom }}\left(\mu_{0}, \mu_{v}, \phi\right) \\
+k_{2}(\lambda) f_{\text {vol }}\left(\mu_{0}, \mu_{v}, \phi\right),
\end{gathered}
$$

where $\mu_{0}$ and $\mu_{\mathrm{v}}$ respectively represent the cosine of solar zenith angles and viewing zenith angle; $f_{\text {iso }}, f_{\text {geom }}$ and $f_{\text {vol }}$ respectively corresponds to the isotropic, geometric-optical (Lisparse kernel) and volumetric (Ross-thick kernel) surface scattering, $f_{\text {iso }}(\lambda) ; k_{1}(\lambda)$ and $k_{2}(\lambda)$ are the coefficients of the BRDF kernels at the wavelength $\lambda$ (Litvinov et al., 2011). For the surface polarized reflectance, the used BPDF is

$$
R_{p}^{S}\left(\mu_{0}, \mu_{\mathrm{v}}, \varphi\right)=\frac{C \exp (\tan \gamma) \exp (\mathrm{NDVI})}{4\left(\mu_{0}+\mu_{\mathrm{v}}\right)} \mathbf{F}_{1,2}\left(\gamma, n_{i}\right),
$$

where $\mathbf{F}_{1,2}\left(\gamma, n_{i}\right)$ represent the polarized component of the Fresnel reflection matrix, $n_{i}$ is the reflective index of the vegetative matter, $\gamma$ means the half the phase angle, NDVI is the normalized difference vegetation index, and $C$ is the only free linear parameter (Maignan et al., 2009). In this paper, he parameters of the BRDF and BPDF of vegetated surface are chosen from the work of Litvinov et al. (2011), which will not be discussed here anymore (Hou et al., 2019).

\begin{tabular}{cl}
\hline No. & Used bands combination \\
\hline 1 & $410 \mathrm{~nm}$ \\
2 & $410,443 \mathrm{~nm}$ \\
3 & $410,443,490 \mathrm{~nm}$ \\
4 & $410,443,490,555 \mathrm{~nm}$ \\
5 & $410,443,490,555,670 \mathrm{~nm}$ \\
6 & $410,443,490,555,670,865 \mathrm{~nm}$ \\
\hline
\end{tabular}

Table 1 . Number of used bands combination for sensitivity study of ALH with the multi-viewing intensity and polarization measurements

In order to obtain the synthetic data of multiangle for sensitivity study, 2 typical multi-viewing observation geometries are considered with the combinations of different solar zenith angles $\left(\theta_{0}\right)$, viewing zenith angles $\left(\theta_{\mathrm{v}}\right)$ and relative azimuth angles $(\phi)$ to represent the observations in different location. Figure 3 illustrates the polar-plot observational geometries, in which the radius corresponds to the $\theta_{\mathrm{v}}$ change from $0^{\circ}$ to $60^{\circ}$ with the step of $20^{\circ}$, and the polar angle means $\phi$ change from $0^{\circ}$ to $360^{\circ}$, as well as the position of Sun also defined with $\phi=0^{\circ}$ (Zheng et al., 2019a). Here, the geometry $\phi=0^{\circ}$ means the observer and Sun are in the same direction and also in the same side of main plane, while $\phi=180^{\circ}$ represents the opposite direction and side of main plane, so as the definition in other polar-plot figures.

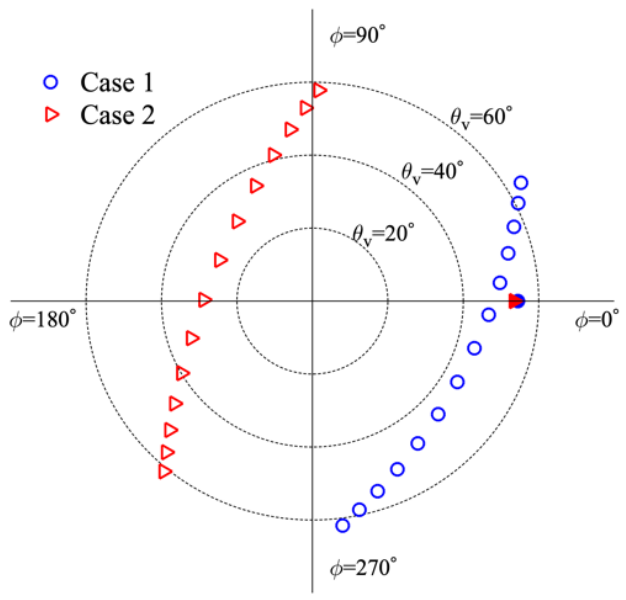

Figure 3. Multi-viewing observation geometries for forward simulations for 2 different cases. In which the solid circle and triangle with $\phi=0^{\circ}$ represent the corresponding position of the Sun for case 1 and case 2 , respectively.

For the sensitivity study of ALH, Table 1 lists the number of used bands combination with the multi-viewing intensity and polarization measurements, in which No.1 means only the 410 nm wavelength is used, and then add the next VIS wavelength in sequence until all of the 6 wavelength bands are used from 410 to $865 \mathrm{~nm}$. Figure 4 illustrates the angular distribution of BRDF results in $410 \mathrm{~nm}$ and the angular distribution of wavelengthindependent BPDF results for the vegetated surface. The angular distribution of BRDF results for other bands are not shown here.

For the aerosol vertical profile parameter settings in UNL-VRTM, the exponentially decreasing profile with scale height $H$ given is used (Wang et al., 2014; Xu and Wang, 2017), which can be written in the form as

$$
\int_{T O A}^{z} \tau(z) d z=\tau_{0} \exp \left(-\frac{z}{H}\right),
$$

where $\tau_{0}$ is the columnar aerosol optical depth (AOD), and we set the scale height $H$ is equal to $2 \mathrm{~km}$ in this study. Besides, we 
set the other aerosol parameters of particle size distribution and complex refractive index with fine-dominated aerosols and AOD $=0.8$ at $550 \mathrm{~nm}$ by following the work of Hou et al. $(2017,2018)$.
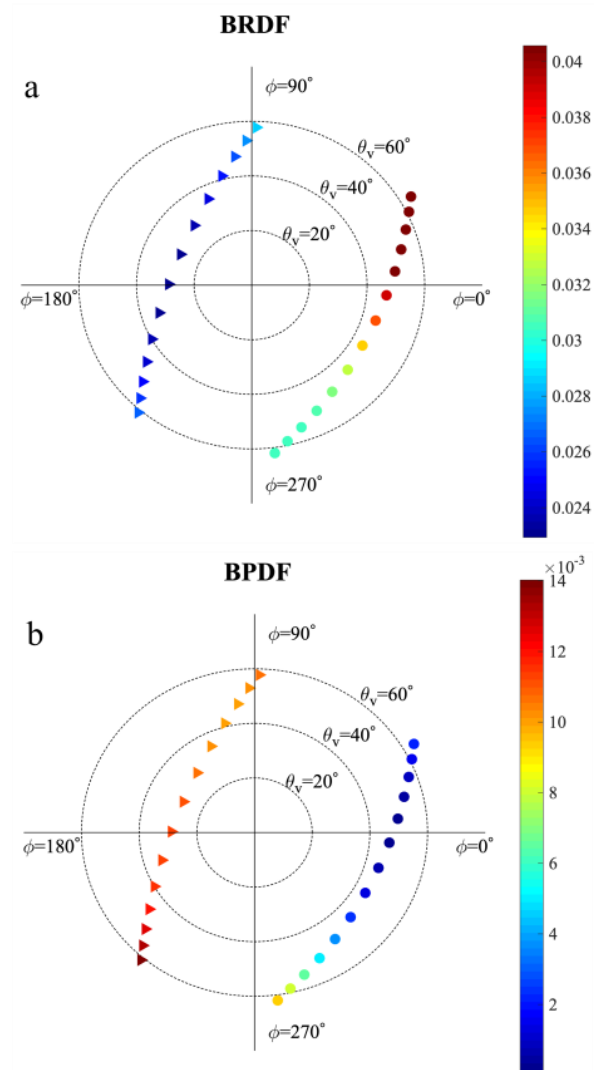

Figure 4. The angular distribution of BRDF results in $410 \mathrm{~nm}$ (a) and the angular distribution of wavelength-independent BPDF results (b) with each colour bar for vegetated surface.

For the information content analysis of ALH in the near-UV and VIS wavelength bands, the state vector only contains one parameter, that is

$$
\mathbf{x}=[H]^{T},
$$

with the vector

$$
\mathbf{b}=\left[V_{0}^{\mathrm{f}}, V_{0}^{\mathrm{c}}, \rho_{s}(\lambda)\right]^{T},
$$

where $V_{0}^{\mathrm{f}}$ and $V_{0}^{\mathrm{c}}$ represents the fine-mode and coarse-mode columnar volume concentration respectively, $\rho_{s}(\lambda)$ is the surface BRDF at the wavelength $\lambda$. The measurement vector $\mathbf{y}$ can be defined as

$$
\mathbf{y}=\left[I_{\lambda}^{v_{1}}, \cdots, I_{\lambda}^{v_{M}}, Q_{\lambda}^{v_{1}}, \cdots, Q_{\lambda}^{v_{M}}, U_{\lambda}^{v_{1}}, \cdots, U_{\lambda}^{v_{M}}\right]^{T},
$$

in which, $I, Q$ and $U$ are the TOA measured Stokes elements, the superscript $v_{1}-v_{M}$ are used to note each viewing in multiple observation. Correspondingly, the Jacobian vector and matrix

$$
\mathbf{K}=\left[\frac{\partial I_{\lambda}^{v_{1}}}{\partial H}, \cdots, \frac{\partial I_{\lambda}^{v_{M}}}{\partial H}, \frac{\partial Q_{\lambda}^{v_{1}}}{\partial H}, \cdots, \frac{\partial Q_{\lambda}^{v_{M}}}{\partial H}, \frac{\partial U_{\lambda}^{v_{1}}}{\partial H}, \cdots, \frac{\partial U_{\lambda}^{v_{M}}}{\partial H}\right]^{T},
$$

with

$$
\mathbf{K}_{\mathbf{b}}=\left[\begin{array}{ccc}
\frac{\partial I_{\lambda}^{v_{1}}}{\partial V_{0}^{\mathrm{f}}} & \frac{\partial I_{\lambda}^{v_{1}}}{\partial V_{0}^{\mathrm{c}}} & \frac{\partial I_{\lambda}^{v_{1}}}{\partial \rho_{s}(\lambda)} \\
\vdots & \vdots & \vdots \\
\frac{\partial I_{\lambda}^{v_{M}}}{\partial V_{0}^{\mathrm{f}}} & \frac{\partial U_{\lambda}^{v_{M}}}{\partial V_{0}^{\mathrm{c}}} & \frac{\partial I_{\lambda}^{v_{M}}}{\partial \rho_{s}(\lambda)}
\end{array}\right] .
$$

For the error covariance matrix

$$
\mathbf{S}_{\mathbf{a}}=\left[\sigma_{H}^{2}\right],
$$

here we set $\sigma_{H}=75 \% H$ with $1.5 \mathrm{~km}$, and

$$
\mathbf{S}_{\mathbf{b}}=\operatorname{diag}\left(\left[\left(\Delta V_{0}^{\mathrm{f}}\right)^{2},\left(\Delta V_{0}^{\mathrm{c}}\right)^{2},\left(\Delta \rho_{s}(\lambda)\right)^{2}\right]^{T}\right),
$$

with prior error of $20 \%$ for $V_{0}^{\mathrm{f}}$ and $V_{0}^{\mathrm{c}}$, and $10 \%$ for $\rho_{s}(\lambda)$. For the measurement error covariance matrix, we have

$$
\mathbf{S}_{\mathbf{y}}=\operatorname{diag}\left(\left[\left(\Delta I_{\lambda}^{v_{1}}\right)^{2}, \cdots,\left(\Delta I_{\lambda}^{v_{M}}\right)^{2}, \cdots,\left(\Delta U_{\lambda}^{v_{1}}\right)^{2}, \cdots,\left(\Delta U_{\lambda}^{v_{M}}\right)^{2}\right]^{T}\right) .
$$

By considering the integrated effects of instrumental noise with radiometric calibration and polarimetric accuracy uncertainties, we assume that the errors of measurements are $5 \%$ for both the intensity and the polarization for the sensitivity study ( $\mathrm{Li}$ et al., 2018)

\section{RESULTS}

\subsection{Simulated TOA Results}
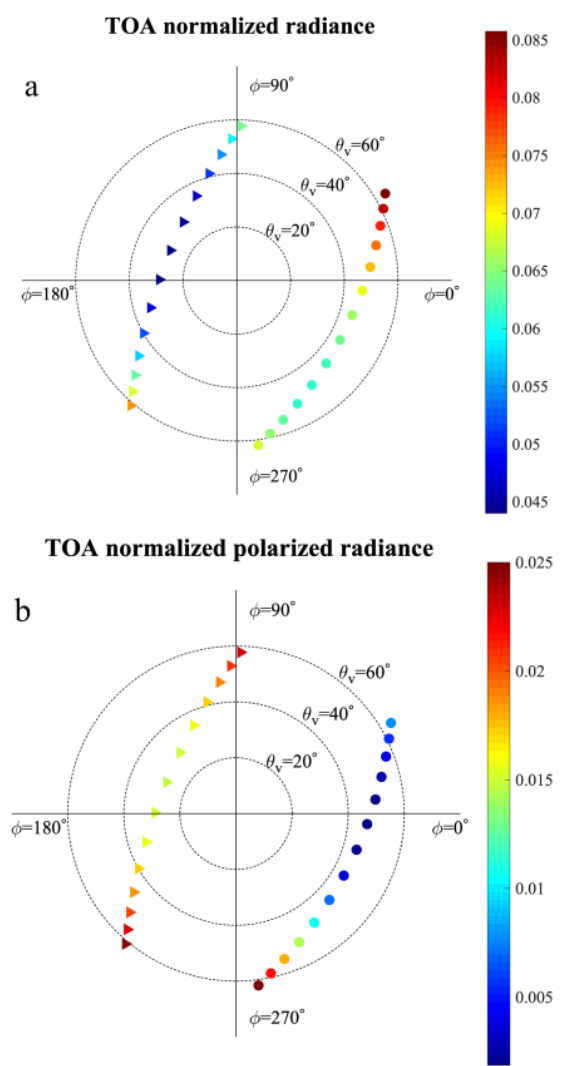

Figure 5. The angular distribution of TOA normalized radiance (a) and normalized polarized radiance (b) in $410 \mathrm{~nm}$ with vegetated surface and $A O D=0.8$ at $550 \mathrm{~nm}$. 
To obtain the synthetic data for the sensitivity study of ALH, 2 multi-viewing observation cases are considered, and the TOA synthetic data are calculated using UNL-VRTM with the vegetated surface and fine-dominated model, in which the aerosols are mixed by the fine- and coarse-mode particles with the fine-mode fraction of columnar volume concentration $\mathrm{FMF}_{V}$ $=0.8$ (Hou et al., 2017, 2018). Figure 5 shows the angular distribution of TOA normalized radiance and polarized radiance in $410 \mathrm{~nm}$. Here, the normalized radiance is equal to the first Stokes element $I$, while the normalized polarized radiance is calculated by $\sqrt{Q^{2}+U^{2}}$. Figure 6 shows the angular distribution of Jacobians of TOA Stokes elements $(I, Q$ and $U$ ) with respect to the aerosol parameters $V_{0}^{\mathrm{f}}$ and $H$ the in $410 \mathrm{~nm}$, respectively. Due to limited space, the angular results in other wavelengths are not shown here.

\subsection{Sensitivity Study Results}

For the DFS and posterior errors of each used bands combination listed in Table 1, we consider the multi-viewing measurements of only intensity and combined with polarization together respectively, and then calculate the mean results of 2 multiviewing observation cases as the final results.
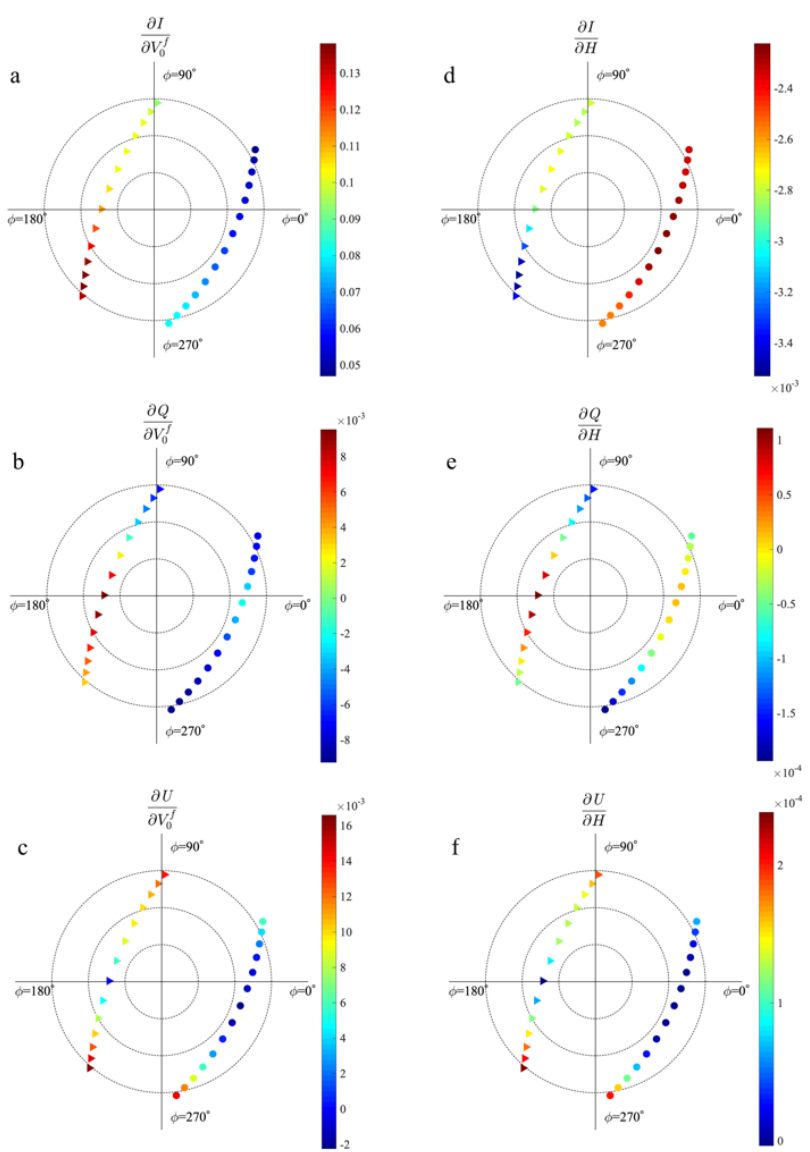

Figure 6. The angular distribution of Jacobians of TOA Stokes elements $I, Q$ and $U$ with respect to $V_{0}^{\mathrm{f}}(\mathrm{a}-\mathrm{c})$ and $H$ (d-f) the in $410 \mathrm{~nm}$.

Figure 7 shows the DPS of ALH along the number of used bands presented in the format of histogram. For the measurements of intensity, the DFS of ALH increases from 0.59 to 0.62 with the increasing number of used bands from 1 to 6 in the range of 410$865 \mathrm{~nm}$ in sequence. While combining the measurements of polarization with intensity together, the information content of
ALH has a significant improvement, and the DFS of ALH increase from 0.94 to 0.98 .

Figure 8 illustrates the posterior errors of ALH along the number of used bands with $75 \%$ priori errors of AHL (absolute priori errors $1.5 \mathrm{~km}$ ). When only the intensity measurements are used, the absolute posterior error decreases form $0.64 \mathrm{~km}$ to $0.61 \mathrm{~km}$ in the range of $410-865 \mathrm{~nm}$ in in sequence. After the polarization measurements are added, the absolute posterior error can decrease from $0.38 \mathrm{~km}$ to $0.21 \mathrm{~km}$. That is to say, the polarization can provide more useful information and constraints for the retrieval of ALH from multi-viewing measurements.

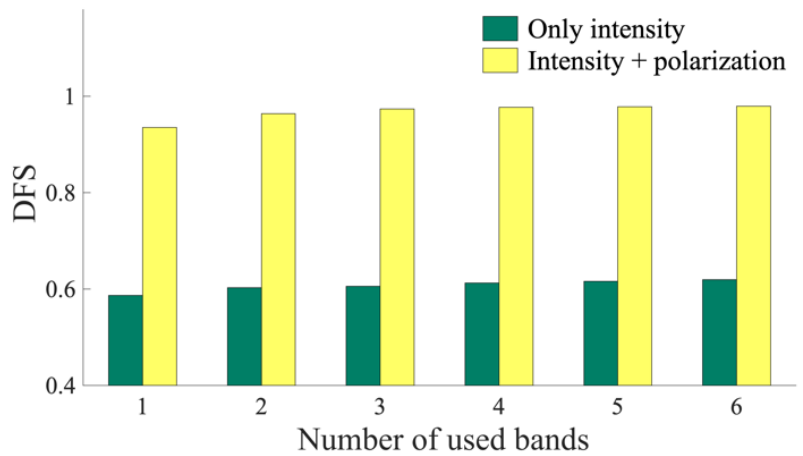

Figure 7. The DPS of ALH along the number of used bands for "only intensity" and "intensity + polarization" multi-viewing measurements.

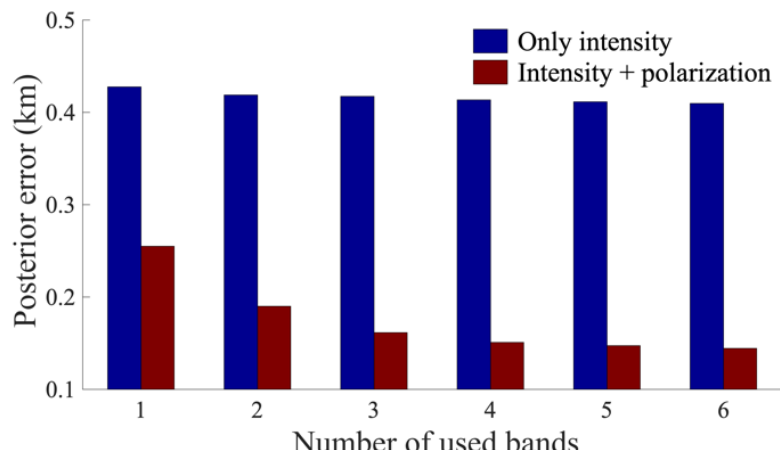

Figure 8. The posterior errors of ALH along the number of used bands for "only intensity" and "intensity + polarization" multi-

viewing measurements with $75 \%$ priori errors of ALH.

\section{CONCLUSIONS}

In the paper, based on the $\mathrm{OE}$ theory and information content analysis method, the sensitivity study of aerosol layer height is carried out with the synthetic data in the range of 410-865 nm, and the capability of multiangle intensity and polarization measurements for the retrieval of ALH has been preliminarily assessed. UNL-VRTM is used as the forward model to calculate the normalized radiance and polarized radiance at the top of atmosphere (TOA), as well as the Jacobians of TOA results with respective to corresponding parameters. The degree of freedom for signal (DFS) and a posteriori error are introduced to quantity the information content of ALH from the intensity and polarization measurements, respectively. By assuming the vegetated surface type, fine-dominated aerosol model, aerosol loads, prior errors and 2 typical multiple observation geometry cases, the preliminarily sensitivity study results show that the near-UV and polarization measurements are the important source of information content for the aerosol height retrieval in satellite remote sensing. 


\section{ACKNOWLEDGEMENTS}

This study was supported by the National Natural Science Foundation of China (Grant Nos. 41871269, 41601385, 41671367, 41505022), and the Open Fund of State Key Laboratory of Remote Sensing Science (Grant No. OFSLRSS201710). We thank the UNL-VRTM codes developed by Prof. Jun Wang in the University of Iowa and Dr. Xiaoguang $\mathrm{Xu}$ in University of Maryland, Baltimore County. The codes used for forward simulations are available at the link of UNL-VRTM (www.unl-vrtm.org).

\section{REFERENCES}

Bi, J., Huang, J., Holben, B., and Zhang, G., 2016. Comparison of key absorption and optical properties between pure and transported anthropogenic dust over East and Central Asia, Atmospheric Chemistry and Physics, 16, 15501-15516.

Butz, A., Guerlet, S., Hasekamp, O., et al., 2011. Toward accurate $\mathrm{CO}_{2}$ and $\mathrm{CH}_{4}$ observations from GOSAT, Geophysical Research Letters, 38(14), L14812.

Chen, X., Wang, J., Liu, Y., et al., 2017. Angular dependence of aerosol information content in CAPI/TanSat observation over land: Effect of polarization and synergy with a-train satellites. Remote Sensing of Environment, 196, 163-177.

Collaud, C.M., Praz, C., Haefele, A., et al., 2014. Determination and climatology of the planetary boundary layer height above the Swiss plateau by in situ and remote sensing measurements as well as by the COSMO-2 model. Atmospheric Chemistry and Physics, $14(23), 13205-13221$

Deuzé, J.L., Bréon, F.M., Devaux, C., et al., 2001. Remote sensing of aerosols over land surfaces from polder-adeos-1 polarized measurements. Journal of Geophysical Research, 106, 4913-4926.

Ding, S., Wang, J., Xu, X., 2016. Polarimetric remote sensing in oxygen A and B bands: sensitivity study and information content analysis for vertical profile of aerosols. Atmospheric Measurement Techniques, 9, 2077-2092.

Dubovik, O., Herman, M., Holdak, A., et al., 2011. Statistically optimized inversion algorithm for enhanced retrieval of aerosol properties from spectral multi-angle polarimetric satellite observations. Atmospheric Measurement Techniques, 4, 9751018 .

Fernald F.G., 1984. Analysis of atmospheric LIDAR observations: Some comments. Applied Optics, 23(5), 652.

Frankenberg, C., Hasekamp, O., O'Dell, C., et al., 2012. Aerosol information content analysis of multi-angle high spectral resolution measurements and its benefit for high accuracy greenhouse gas retrievals. Atmospheric Measurement Techniques, 5, 1809-1821.

Hasekamp, O.P., Litvinov, P., Butz, A., 2011. Aerosol properties over the ocean from PARASOL multiangle photopolarimetric measurements. Journal of Geophysical Research, 116, D14204.

Haywood, J., Boucher, O., 2000. Estimates of the direct and indirect radiative forcing due to tropospheric aerosols: A review. Reviews of Geophysics, 38(4), 513-543.
Hou, W., Wang, J., Xu, X., et al., 2016. An algorithm for hyperspectral remote sensing of aerosols: 1 . Development of theoretical framework. Journal of Quantitative Spectroscopy and Radiative Transfer, 178, 400-415.

Hou, W., Wang, J., Xu, X., et al., 2017. An algorithm for hyperspectral remote sensing of aerosols: 2 . Information content analysis for aerosol parameters and principal components of surface spectra. Journal of Quantitative Spectroscopy and Radiative Transfer, 192, 14-29.

Hou, W., Li, Z., Wang, J., et al., 2018a. Improving remote sensing of aerosol microphysical properties by near-infrared polarimetric measurements over vegetated land: Information content analysis. Journal of Geophysical Research: Atmospheres, $123,2215-2243$.

Hou, W., Li, Z., Zheng, F., et al., 2018b. Retrieval of aerosol microphysical properties based on the optimal estimation method: Information content analysis for satellite polarimetric remote sensing measurements. Int. Arch. Photogramm. Remote Sens. Spatial Inf. Sci., ISPRS TC III Mid-term Symposium, 533-537.

Hou, W., Mao, Y., Xu, C., et al., 2019. Study on the spectral reconstruction of typical surface types based on spectral library and principal component analysis. Proc. SPIE, 11023, 110232T

Gamage, N., and Hagelberg, C., 1993. Detection and analysis of microfronts and associated coherent events using localized transforms. Journal of the Atmospheric Sciences, 50, 750-756.

Gordon, H.R., 1997. Atmospheric correction of ocean color imagery in the Earth Observing System era. Journal of Geophysical Research: Atmospheres, 102, 17081-17106

Gu, X., Tong, X., 2015. Overview of China earth observation satellite programs. IEEE Geoscience and Remote Sensing Magazine, 3, 113-129.

Li, Z. Q., Guo, J. P., Ding, A. J., et al., 2017. Aerosol and boundary-layer interactions and impact on air quality. National Science Review, 4(6), 810-833.

Li, Z., Hou, W., Hong, J., et al., 2018. Directional Polarimetric Camera (DPC): Monitoring aerosol spectral optical properties over land from satellite observation. Journal of Quantitative Spectroscopy and Radiative Transfer, 218, 21-37.

Liu, Z., Mortier, A., Li, Z., et al., 2017. Improving daytime planetary boundary layer height determination from CALIOP: Validation based on ground-based lidar station. Advances in Meteorology, 5759074, 1-14

Litvinov, P., Hasekamp, O., Cairns, B., 2011. Models for surface reflection of radiance and polarized radiance: Comparison with airborne multi-angle photopolarimetric measurements and implications for modeling top-of-atmosphere measurements. Remote Sensing of Environment, 115, 781-792.

Maignan, F., Bréon, F.-M., Fédèle, E., et al., 2009. Polarized reflectances of natural surfaces: Spaceborne measurements and analytical modeling. Remote Sensing of Environment, 113, 26422650.

Marbach, T., Phillips, P., Lacan, A., et al., 2013. The MultiViewing, -Channel, -Polarisation Imager (3MI) of the 
EUMETSAT Polar System - Second Generation (EPS-SG) dedicated to aerosol characterisation. Proc. of SPIE, 88890I1-9.

McGill, M. J., Hlavka, D. L., Hart, W. D., 2003. Airborne lidar measurements of aerosol optical properties during SAFARI-2000, Journal of Geophysical Research, 108(D13), 8493.

Mishchenko, M.I., Cairns, B., Hansen, J.E., et al., 2007. Accurate monitoring of terrestrial aerosols and total solar irradiance: Introducing the glory mission. Bulletin of the American Meteorological Society, 88, 677-691.

Rodgers, C.D., 2000. Inverse methods for atmospheric sounding: Theory and practice. World Scientific, Singapore, 13-40.

Sanders, A.F.J., de Haan, J.F., Sneep, M., et al., 2015. Evaluation of the operational aerosol layer height retrieval algorithm for Sentinel-5 Precursor: application to $\mathrm{O}_{2} \mathrm{~A}$ band observations from GOME-2A. Atmospheric Measurement Techniques, 8, 49474977.

Sanghavi, S., Martonchik, J.V., Landgraf, J., et al., 2012. Retrieval of the optical depth and vertical distribution of particulate scatterers in the atmosphere using $\mathrm{O}_{2} \mathrm{~A}$ - and B-band SCIAMACHY observations over Kanpur: a case study. Atmospheric Measurement Techniques, 5, 1099-1119.

Seibert, P., Beyrich, F., Gryning, S.-E., et al., 2000. Review and intercomparison of operational methods for the determination of the mixing height, Atmospheric Environment, 34(7), 1001-1027.

Spurr, R., 2006. Vlidort: A linearized pseudo-spherical vector discrete ordinate radiative transfer code for forward model and retrieval studies in multilayer multiple scattering media. Journal of Quantitative Spectroscopy and Radiative Transfer, 102, 316342 .

Tucker, S. C., Senff, C. J., Weickmann, A. M., et al., 2009. Doppler lidar estimation of mixing height using turbulence, shear, and aerosol profiles, Journal of Atmospheric and Oceanic Technology, 26, 673-688.

Wang, H., Li, Z., Lv, Y., et al., 2019. Observational study of aerosol-induced impact on planetary boundary layer based on lidar and sunphotometer in Beijing, Environmental Pollution, 252, 897-906.

Wang, J., and Christopher, S.A., 2003. Intercomparison between satellite-derived aerosol optical thickness and PM2.5 mass: implications for air quality studies. Geophysical Research Letters, 30, 2095, 1-4.

Wang, J., Xu, X., Ding, S., et al., 2014. A numerical testbed for remote sensing of aerosols, and its demonstration for evaluating retrieval synergy from a geostationary satellite constellation of geo-cape and goes-r. Journal of Quantitative Spectroscopy and Radiative Transfer, 146, 510-528.

Waquet, F., Cairns, B., Knobelspiesse, K., et al., 2009. Polarimetric remote sensing of aerosols over land. Journal of Geophysical Research, 114, D01206.

Wiegner, M., Madonna, F., Binietoglou, I., et al., 2014. What is the benefit of ceilometers for aerosol remote sensing? An answer from EARLINET, Atmospheric Measurement Techniques, 7(7), 1979-1997.
Winker, D.M., Pelon, J., Coakley, J.A., et al., 2010. The CALIPSO mission. Bulletin of the American Meteorological Society, 91, 1211-1230

Wu, L., Hasekamp, O., van Diedenhoven, B., et al., 2015. Aerosol retrieval from multiangle, multispectral photopolarimetric measurements: Importance of spectral range and angular resolution. Atmospheric Measurement Techniques, 8, 2625-2638.

Wu, L., Hasekamp, O., van Diedenhoven, B., et al., 2016. Passive remote sensing of aerosol layer height using near-UV multiangle polarization measurements. Geophysical Research Letters, 43, 8783-8790.

Xu, X., Wang, J., 2015. Retrieval of aerosol microphysical properties from Aeronet photopolarimetric measurements: 1. Information content analysis. Journal of Geophysical Research: Atmospheres, 120, 7059-7078.

Xu, X., Wang, J., Zeng, J., et al., Siniuk, A., Holben, B. N., 2015. Retrieval of aerosol microphysical properties from aeronet photopolarimetric measurements: 2. A new research algorithm and case demonstration. Journal of Geophysical Research: Atmospheres, 120, 7079-7098.

Xu, X., Wang, J., Wang, Y., et al., 2017a. Sense size-dependent dust loading and emission from space using reflected solar and infrared spectral measurements: An observation system simulation experiment. Journal of Geophysical Research: Atmospheres, 122, 8233-8254.

Xu, X., Wang, J., Wang, Y., et al., 2017b. Passive remote sensing of altitude and optical depth of dust plumes using the oxygen a and b bands: First results from epic/dscovr at lagrange-1 point. Geophysical Research Letters, 44, 7544-7554.

Xu, X., and Wang, J., 2017. User's Guide for UNL-VRTM: A UNified Linearized Vector Radiative Transfer Model, Version 1.6.2, The University of Iowa.

Xu, X., Wang, J., Zeng, J., et al., 2018. A pilot study of shortwave spectral fingerprints of smoke aerosols above liquid clouds. Journal of Quantitative Spectroscopy and Radiative Transfer, 221, 38-50.

Xu, X., Wang, J., Wang, Y., et al., 2019. Detecting layer height of smoke aerosols over vegetated land and water surfaces via oxygen absorption bands: hourly results from EPIC/DSCOVR in deep space. Atmospheric Measurement Techniques, 12, 32693288.

Yang, D., Li, C., Lau, A.K.H., et al., 2013. Longterm measurement of daytime atmospheric mixing layer height over Hong Kong, Journal of Geophysical Research: Atmospheres, $118,2422-2433$.

Zheng, F., Hou, W., Li, Z., 2019a. Optimal estimation retrieval for directional polarimetric camera onboard Chinese Gaofen-5 satellite: an analysis on multi-angle dependence and a posteriori error. Acta Phys. Sin., 68, 040701.

Zheng, F., Hou, W., Sun, X., et al., 2019b. Optimal estimation retrieval of aerosol fine-mode fraction from ground-based sky light measurements. Atmosphere, 10, 1-16. 